\title{
Low Doses of Nonylphenol Promote Growth of Colon Cancer Cells through Activation of ERK1/2 via G Protein-Coupled Receptor 30
}

\section{Ming Xie, BA \\ Jin-Long Liang, MM \\ Han-Dong Huang, MM \\ Mai-Jian Wang, MM \\ Tao Zhang, MM \\ Xue-Feng Yang, MM}

Department of Gastrointestinal Surgery, Affiliated Hospital of Zunyi Medical College, Zunyi, China

\begin{abstract}
Purpose
Nonylphenol (NP) is an endocrine disruptor found in products such as cleaners, plastics, and detergents. It exerts actions similar to endogenous 17ß-estradiol (E2) and is reported to influence various cancers. However, its role in colon cancer remains elusive.
\end{abstract}

\section{Materials and Methods}

Colon cancer cell lines COLO 205 and SW480 were employed in our study. The cells were treated with NP or E2 followed by measurement of apoptosis and proliferation using flow cytometry and MTT assays, respectively. G protein-coupled estrogen receptor 30 (GPR30) expression was visualized using immunofluorescence and Western blot. To investigate the underlying mechanism, the expression levels of GPR30, p-protein kinase A (PKA), c-myc, cyclin D1, and ERK1/2 were analyzed using Western blot. Meanwhile, the GPR30 antagonist G15 was utilized to validate the role of GPR30 in colon cancer progression. Finally, the effect of a GPR30 inhibitor on tumor growth was determined in vivo using tumor xenograft mouse models.

\section{Results}

NP facilitated the proliferation of colon cancer cells and induced apoptosis failure in vitro. Western blot revealed increased GPR30 expression levels in response to NP treatment. Cyclin D1, p-PKA, c-myc, and proliferating cell nuclear antigen, proteins that regulate the cell cycle, were all upregulated by NP, and NP-mediated ERK1/2 activation and subsequent cell proliferation were abrogated by the GPR30 inhibitor G15. Moreover, colon cancer mice that received G15 administration demonstrated impaired tumor growth in vivo.

\section{Conclusion}

Low dose NP promotes the growth of colon tumors through GPR30-mediated activation of ERK1/2 signaling.
No. 149, Dalian Road, Zunyi 563000, Guizhou

Province, China

Tel: 86-13985200658

Fax: 86-085128622043

E-mail: yangxuefeng923@163.com

Received June 5, 2018

Accepted April 12, 2019

Published Online May 15, 2019

\section{Key words}

Nonylphenol, GPR30, Colon neoplasms, ERK1/2, Cell proliferation, Cyclin D1

\section{Introduction}

Active hormone exposure is common in our lives, for example, from cigarettes, automobile exhaust, and cosmetic products, and the majority of these compounds are xenoestrogens, such as nonylphenol (NP), zearalenone, and bisphenol A. Xenoestrogens demonstrate estrogenic efficacy in the body through competitively binding to estrogen receptors. Estrogens regulate their effects through two estrogen receptors (ERs), ER $\alpha$ and $E R \beta$, which differentially activate two downstream pathways. The estrogen response is tissuespecific and determined by the local ratio of ER $\alpha$ and ER $\beta$. Generally, ERs exert their effects through both genomic and nongenomic mechanisms. In the former pathway, ERs activate gene transcription through interaction with estrogen receptor response elements in DNA. In other cases, ERs modulate diverse cellular pathways independent of interactions with DNA. For example, ER $\alpha$ promotes cell proliferation and survival via phosphoinositide 3-kinase activation, while ER $\beta$ 
reportedly inhibits cell cycle progression through interactions with cyclin D1 [1]. Recent studies demonstrated a seven-transmembrane $\mathrm{G}$ protein-coupled receptor, GPR30, rapidly responds to estrogen through cellular signaling [2]. GPR30 is reported to mediate cellular estrogen functions, including traditional genomic signaling through transcriptional regulation and rapid non-genomic estrogen signaling. The study demonstrated that estrogen activates mitogenactivated protein kinase/ERK1/2 through transactivation of epidermal growth factor receptor [3]. Subsequently, GPR30 was found to mediate estrogen-induced apoptosis through upregulating Bcl-2 expression [4]. Moreover, it was reported that estrogen induces c-fos transcription through ERK1/2 activation via GPR30, promoting the progression of breast cancer [5]. Thus, GPR30 seems to participate in estrogenmediated tumorigenesis and tumor progression, making it a potentially efficient therapeutic target for cancer treatment.

Colorectal cancer, also known as colon cancer, is one of the most common malignant gastrointestinal cancers [6]. The differential incidence rate between men and women suggests a hormonal role in the development of this disease [7]. The role of estrogen and xenoestrogens in cancer development has been intensively studied over the past decades. Bisphenol A reportedly increases the proliferation of human breast cancer cell lines in vitro and induces oxidative stress [8]. In addition, neoplastic transformation is observed in human breast epithelial cells exposed to bisphenol A [9]. It was reported that NP increases tumor formation and growth by inhibiting lymphocyte proliferation and macrophage activation [10]. The study demonstrated that NP enhances the progression of prostate cancer by modulating proteins that regulate the cell cycle, apoptosis, and metastasis, such as cyclin D1, cyclin E, p21, bax, and cathepsin D [11]. However, the effects of NP on colon cancer remain elusive.

Previous studies revealed a close relationship between colon cancer and 17ß-estradiol (E2), the most abundant and most potent estrogen in humans. E2 plays a protective role in the development of colon cancer through ER $\beta$, which is highly expressed in normal colonic mucosa but dramatically reduced in colon cancer [12]. Estrogen replacement therapy is reported to reduce colon cancer risk by $30 \%-40 \%$ in postmenopausal women [13]. It has been suggested that E2 inhibits the initiation and development of colon cancer, which could explain the lower incidence rate of colon cancer in women at all ages compared to men.

In the present study, we report that NP promotes proliferation and induces apoptosis failure in colon cancer cells through increasing expression of GPR30, which subsequently activates ERK1/ 2 signaling. In addition, the GPR30 inhibitor G15 abrogated NP-induced proliferation and inhibited tumor growth in vivo. Our study reveals the pro-tumorigenic role of xenoestrogens in colon cancer and suggests that
GPR30 antagonism could be a novel therapeutic strategy for colon cancer.

\section{Materials and Methods}

\section{Animals}

Twelve-week-old male nude BALB/c mice were purchased and housed at the animal research facility of Zunyi Medical College. Mice were acclimatized to laboratory conditions and provided access to food and water ad libitum. Mice were kept for 7 days before experiments and housed throughout the study in an environment of $50 \pm 10 \%$ relative humidity, $24 \pm 2^{\circ} \mathrm{C}$, and a 12-hour light/dark cycle.

\section{Cell culture and cell treatment}

The human colon cancer cell line SW480 and colorectal adenocarcinoma cell line COLO205 were obtained from American Type Culture Collection and cultured in Dulbecco's modified Eagle's medium (DMEM) and minimum essential medium alpha, respectively. Complete culture medium was supplemented with $10 \%$ fetal bovine serum, $100 \mathrm{U} / \mathrm{mL}$ penicillin and $100 \mu \mathrm{g} / \mathrm{mL}$ streptomycin, and cells were cultured at $37^{\circ} \mathrm{C}$ in a humidified atmosphere with $5 \% \mathrm{CO}_{2}$. All cell culture reagents were purchased from Invitrogen (Carlsbad, CA).

For proliferation and immunocytochemistry assays, cells were divided into three groups: (1) control group, (2) E2 group (cells treated with $10^{-7}, 10^{-6}$, or $10^{-5} \mathrm{M}$ of E2 for 24 hours), (3) NP group (cells treated with $10^{-7}, 10^{-6}$, or $10^{-5} \mathrm{M}$ of $\mathrm{NP}$ for 24 hours). For studying NP's mechanism, cells were divided into three groups: (1) control group, (2) NP group (cells treated with NP for $0,24,48$, or 72 hours), and (3) $\mathrm{NP}+\mathrm{G} 15$ (cells pre-treated with G15 for 90 minutes followed by changing to medium containing NP).

\section{MTT assay}

To assess cell proliferation, 3-(4,5-dimethylthiazol-2-yl)2,5-diphenyl tetrazoliumbromide (MTT) was used. Briefly, cells were seeded in 96-well plates at a final concentration of $2 \times 10^{4}$ cells / well. At the end of treatment, the media was removed, and $20 \mu \mathrm{L}$ MTT dye solution $(5 \mathrm{mg} / \mathrm{mL}$ in phosphate buffered saline [PBS] buffer) was added per well and allowed to incubate at $37^{\circ} \mathrm{C}$ for 4 hours. Then, the supernatant was decanted and replaced with $150 \mu \mathrm{L}$ dimethyl sulfoxide. After 15-minute incubation with gentle shaking at $37^{\circ} \mathrm{C}$, absorbance was measured at a wavelength of $490 \mathrm{~nm}$ 
using a microtiter plate reader (Tecan, Maennedorf, Switzerland).

\section{Flow cytometry for apoptosis analysis}

Flow cytometry was employed to detect apoptosis. After treatment, cells were collected with trypsin digestion solution, washed twice with PBS, and resuspended in $200 \mu \mathrm{L}$ PBS. For apoptosis analysis, the Annexin V/Dead Cell Apoptosis Kit (Invitrogen) was utilized according to the manufacturer's instructions. Briefly, $5 \mu \mathrm{L}$ Annexin V-FITC and $5 \mu \mathrm{L}$ propidium iodide were added to each well, and the cells were incubated in the dark for 15 minutes. Then, apoptosis was measured using a FACScan flow cytometer (Becton, CA) equipped with CellQuest Software (Becton Dickinson).

\section{Immunocytochemistry}

Cells were fixed with $4 \%$ paraformaldehyde, washed with PBS, and permeabilized with $0.1 \%$ Triton X-100 for 5 minutes. Samples were then blocked for 1 hour with $5 \%$ bovine serum albumin in PBS. Then, cells were incubated with rabbit anti-GPR30 (Abcam, Cambridge, MA) primary antibody for 1 hour at room temperature. Specific secondary antibodies conjugated with green fluorescent protein fluorochrome (Invitrogen) were subsequently incubated with cells for 1 hour at room temperature. After PBS washes, slides were mounted. Images were captured using an optical microscope (DP73, Olympus Corp.).

\section{RNA extraction, reverse transcription, and quantitative polymerase chain reaction}

Total RNA was extracted using TRIzol reagent (Invitrogen) according to the manufacturer's instructions, and each sample was reverse transcribed to cDNA using the PrimeScript RT reagent Kit with gDNA Eraser (Takara, Dalian, China). Gene expression of GPR30, proliferating cell nuclear antigen (PCNA), c-myc, and cyclin D1 was measured by real-time quantitative reverse transcription polymerase chain reaction (qRT-PCR) using an Applied Biosystems 7500 Fast RealTime qRT-PCR machine. Polymerase chain reaction primers used for reverse transcription polymerase chain reaction were synthesized by Invitrogen and sequences are as follows: hPCNA216 forward primers, CGGATACCTTGGCGCTAGTA; hPCNA-216 reverse primers, TCACTCCGTCTTTTGCACAG; hc-myc-175 forward primers, GGACTTGTTGCGGAAACGAC; hc-myc-175 reverse primers, CTCAGCCAAGGTTGTGAGGT; hcyclinD1-113 forward primers, AGCTGTGCATCTACACCGAC; hcyclin-D1-113 reverse primers, GAAATCGTGCGGGGTCATTG; hGPR30 forward primers, CTCTTCCCCATCGGCTTTGT; hGPR30 reverse primers, TACAG-
GTCGGGGATGGTCAT; hGAPDH forward primers, CCTCCCGCTTCGCTCTCT; hGAPDH reverse primers, CCGTTGACTCCGACCTTCAC.

\section{Western blotting}

Whole cell extracts were prepared from tissue or cells using RIPA buffer, and protein concentrations were determined using a bicinchoninic acid assay. Equal aliquots of proteins were separated using 8\%-10\% sodium dodecyl sulfate polyacrylamide gel electrophoresis. Following electrophoresis, separated proteins were transferred onto polyvinylidene difluoride membranes (EMD Millipore, Billerica, MA) and blocked with 5\% BSA for 1 hour at room temperature. Membranes were then incubated overnight at $4^{\circ} \mathrm{C}$ with primary antibodies. Primary antibodies used are as follows: anti-GPR30 (ab39742) and anti-GAPDH (ab8245) was purchased from Abcam (Cambridge, UK), anti-ERK1 / 2 (\#9194), and anti-ERK1/ 2 (\#8544), PCNA (\#13110), ani-phosphorylated protein kinase A (pPKA) (\#5661), anti-PKA (\#4782), c-myc (\#5605), and cyclin D1 (\#2978) were purchased from Santa Cruz Biotechnology (Boston, MA). Membranes were then washed with TBST buffer and incubated with horseradish peroxidase-conjugated secondary antibody for 1 hour. Protein bands were visualized using the enhanced chemiluminescence detection system (GE ImageQuantLAS4000, Tokyo, Japan). Band intensity was quantified using Image J software.

\section{Soft agar colony formation assay}

The soft agar colony-forming assay was performed to assess the colony formation and invasion potential of cancer cells. Briefly, 24 hours after treatment, cells were mixed with DMEM containing $0.35 \%$ soft agar (Sigma-Aldrich, St. Louis, $\mathrm{MO}$ ). Then, cells were seeded in 35-mm dishes coated with DMEM containing $0.6 \%$ soft agar. After 10 days, the number of colonies were counted, and images were captured using an optical microscope (DP73, Olympus Corp.).

\section{Tumor xenografts in athymic nude mice}

SW480 cells were suspended in PBS at a final concentration of $1 \times 10^{8}$ cells $/ \mathrm{mL}$. A volume of $0.1 \mathrm{~mL}$ of suspended cells was subcutaneously injected into a single side of the posterior flank of each mouse ( $n=5 /$ group). Five days after cell injection, tumor-bearing mice were randomly divided into three groups ( 6 animals per group): (1) control (no treatment), (2) NP treatment $(80 \mu \mathrm{mol} / \mathrm{kg})$, or (3) NP+G15 treatment (mice were administered $200 \mu \mathrm{g} / \mathrm{kg}$ G15 2 days before sacrifice). Mice were intraperitoneally injected with corn oil at a dose of $10 \mathrm{~mL} / \mathrm{kg}$ for 14 days. At the end of the treat- 

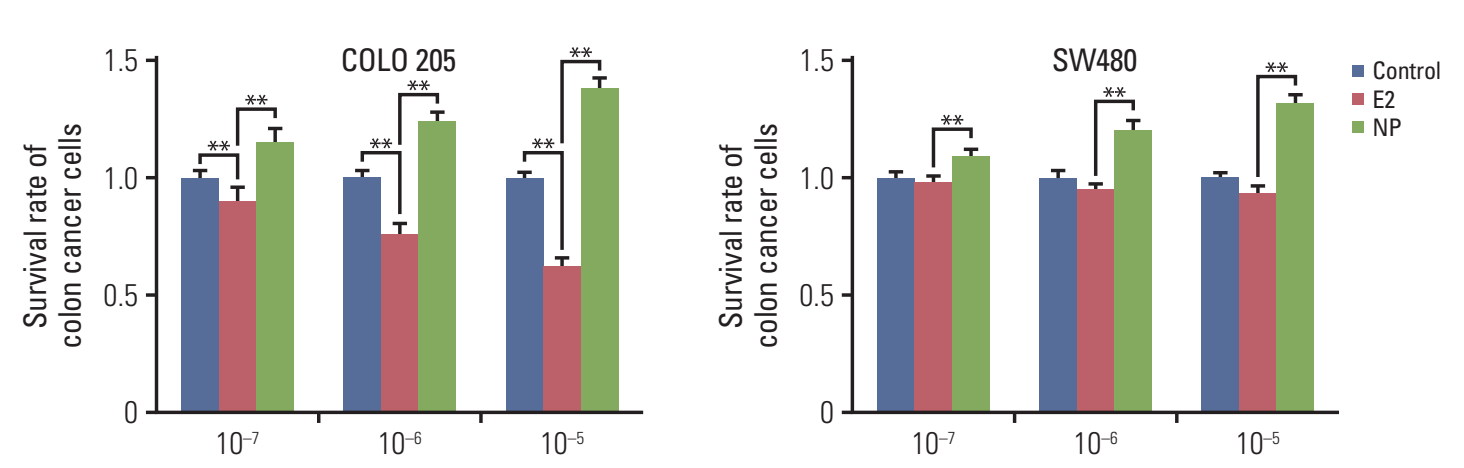

A
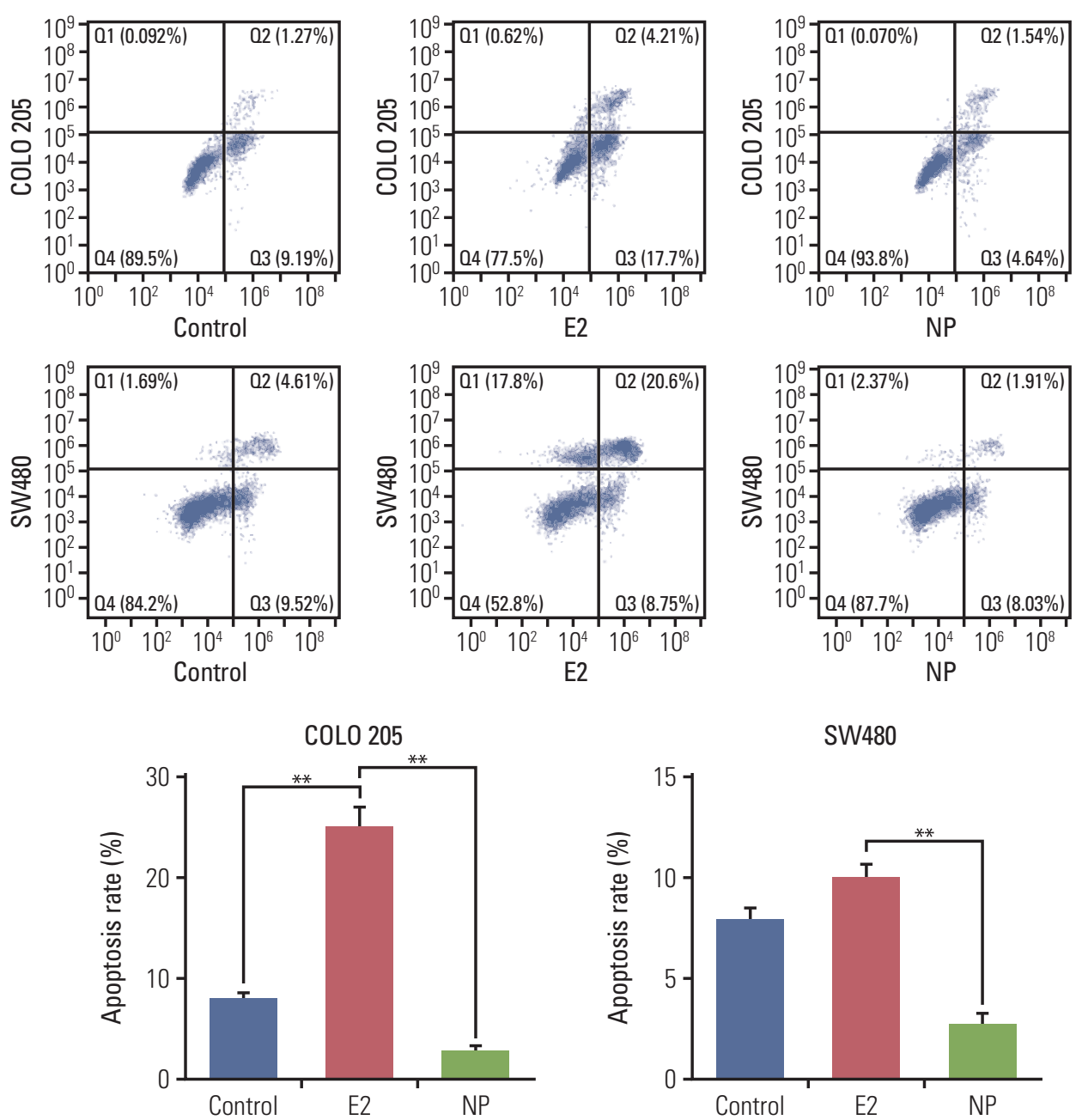

Fig. 1. Nonylphenol (NP) increases cell proliferation and inhibits apoptosis in colon cancer cells. (A) Cell viability detected by MTT assay. (B) Apoptosis analyzed by flow cytometry. $n=3$. Significant difference compared to the control group ${ }^{* *} \mathrm{p}<$ 0.01). E2, 17ß-estradiol. 
ment, animals were sacrificed. Briefly, mice were anaesthetized with isoflurane in a closed chamber followed by exposure to carbon dioxide for ten minutes for euthanasia, and tumors were photographed.

\section{Histology}

Isolated lung tissues were embedded in paraffin and cut into $4 \mu \mathrm{M}$ sections using a rotary microtome (Leica, Mannheim, Germany). Haematoxylin and eosin staining was used to visualize tissue structure. Dried slices were soaked in xylene, dewaxed for 10 minutes, rehydrated, rinsed in hematoxylin for 5 minutes and washed again prior to differentiation with $1 \%$ hydrochloric acid alcohol. Then, slides were stained with 0.55 eosin for 30 seconds, dehydrated with gradient alcohol, and soaked in xylene three times. Finally, slides were mounted using neutral gum. For immunohisto- chemistry staining, slides were stained with antibodies against Ki67 (\#9449, Cell Signaling Technologies, Boston, MA) before hematoxylin co-staining. Histopathological changes of tissues were analyzed using an optical microscope (DP73, Olympus Corp.).

\section{Statistics}

Data are presented as the mean \pm standard error of mean. Statistical analysis between multiple groups was performed using one-way analysis of variance (ANOVA) with Dunnett's post-test. Student's t test was employed to compare differences between the two groups. A two-sided value of $\mathrm{p}<0.05$ was considered statistically significant. All statistical analyses were performed using GraphPad Prism 5 software (GraphPad Software, La Jolla, CA).

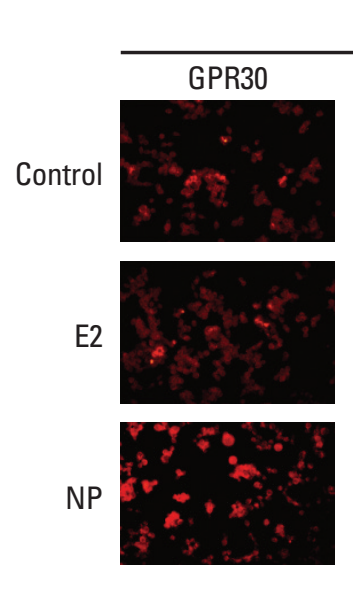

COLO 205
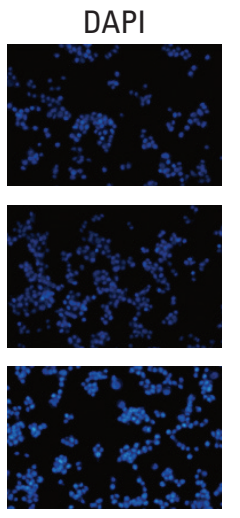
Merge
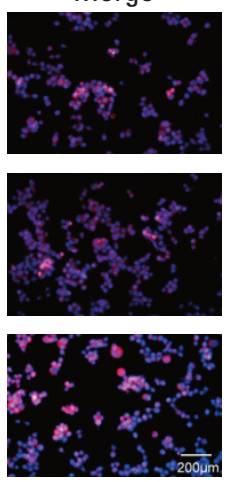

A

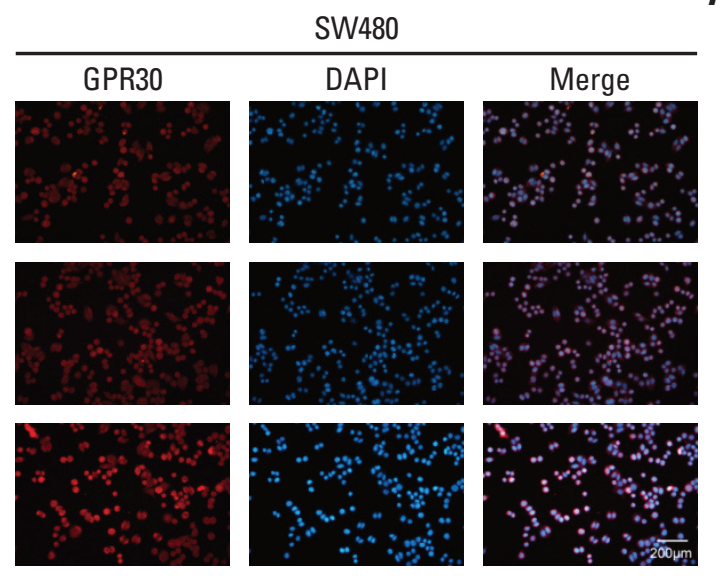

B
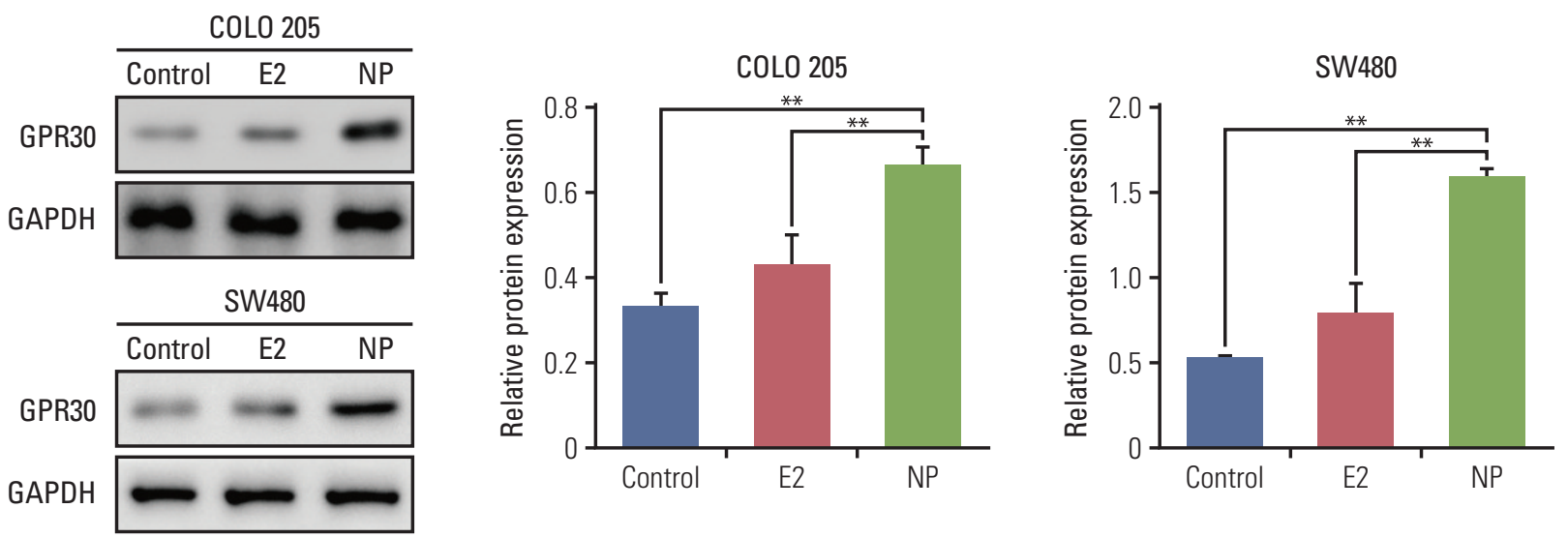

Fig. 2. Nonylphenol (NP) increases expression of G protein-coupled estrogen receptor 30 (GPR30). (A) Immunocytochemistry staining of GPR30 (red) and nuclei (blue) in COLO 205 and SW480 cells. (B) Western blot of GPR30 levels in cells $\left({ }^{* *} \mathrm{p}<0.01\right)$. GAPDH, glyceraldehyde 3-phosphate dehydrogenase. 

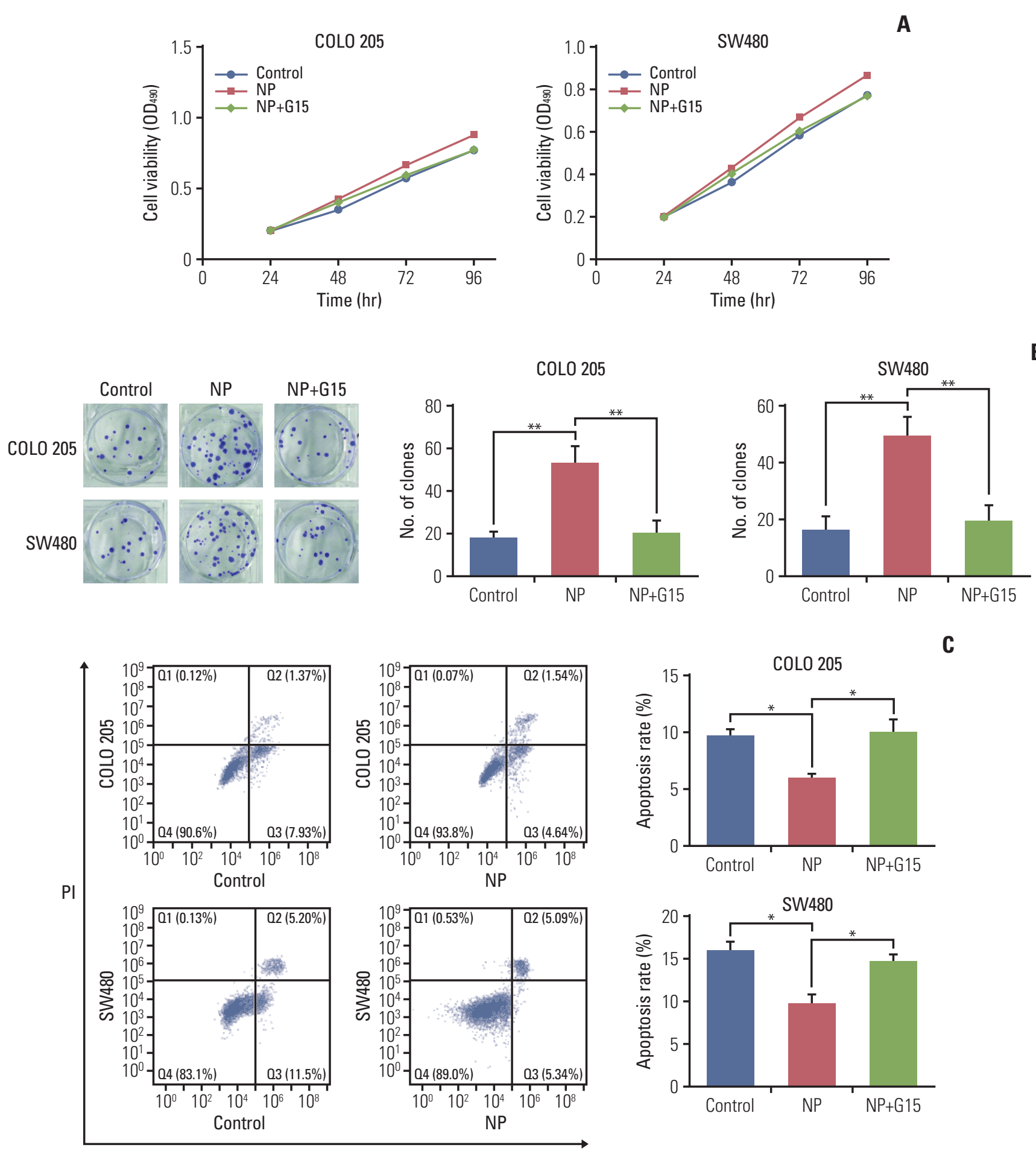

Annexin V

Fig. 3. Nonylphenol (NP) promotes cell proliferation of colon cancer cells through G protein-coupled estrogen receptor 30 (GPR30). (A) Cell viability detected by MTT assay. (B) Colony formation efficiency analyzed using soft agar colony formation assay. (C) Apoptosis analyzed by flow cytometry. $n=3$. Significant difference compared to the control group $\left({ }^{*} \mathrm{p}<0.05\right.$, $\left.{ }^{* *} \mathrm{p}<0.01\right)$. G15, GPR30 inhibitor. 


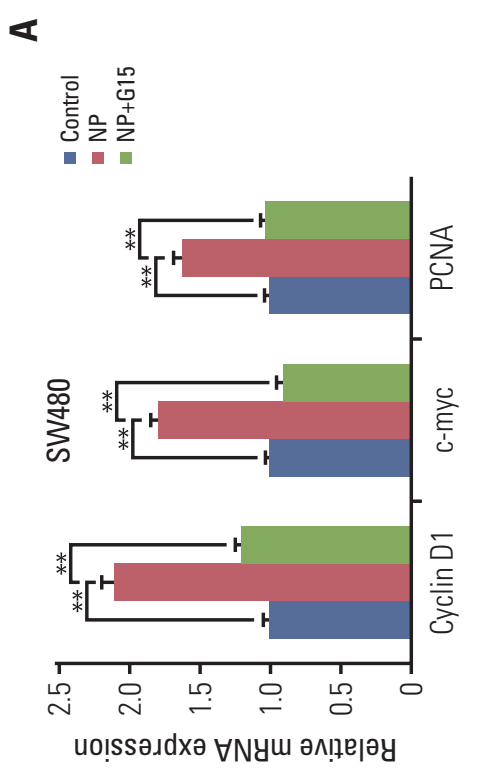

$\infty$
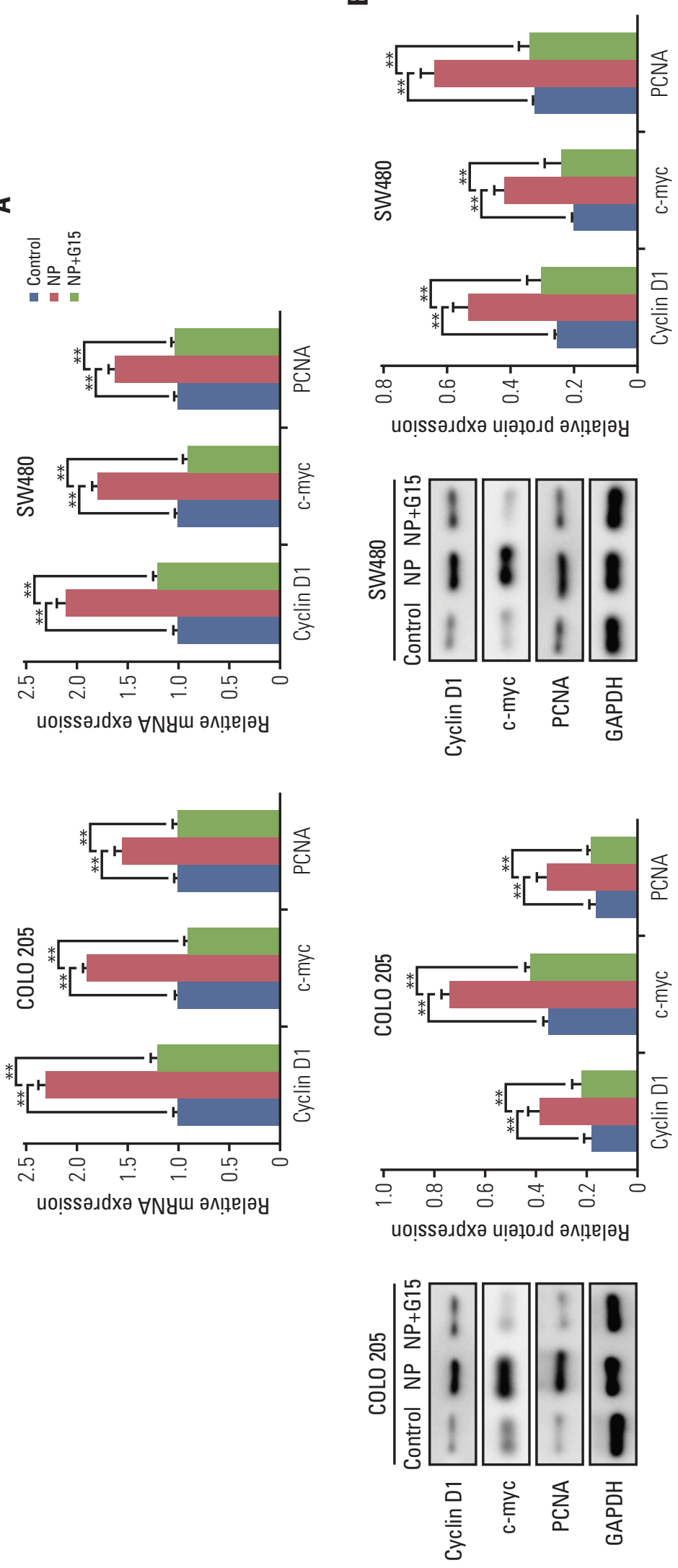

ง

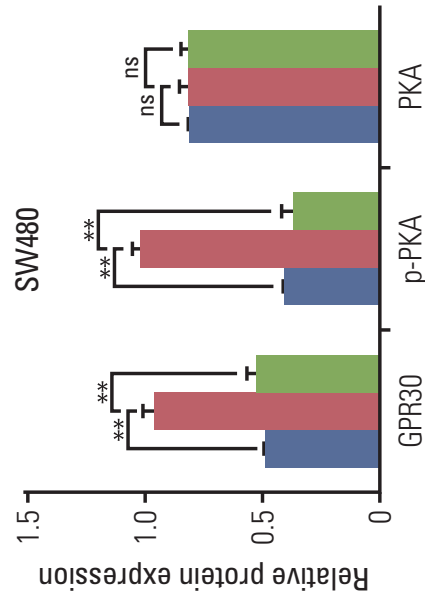

ฮี 串

芑苔式

吉 焉焉

b. E

莺 壳

岕苛

范范

हैं士

u.

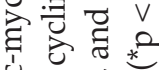

पे

它获

专高

S

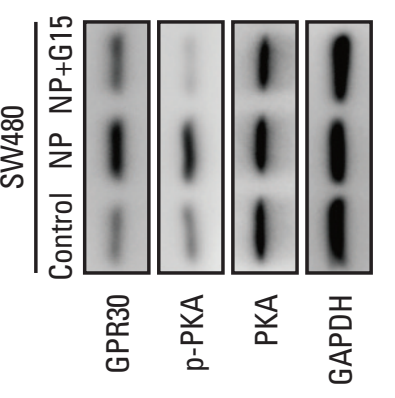

उ范

के

는 몽

艺过

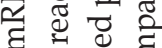

运考

मี

के कृ के

至

㐅े बे

들

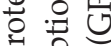

ㄹ. 영

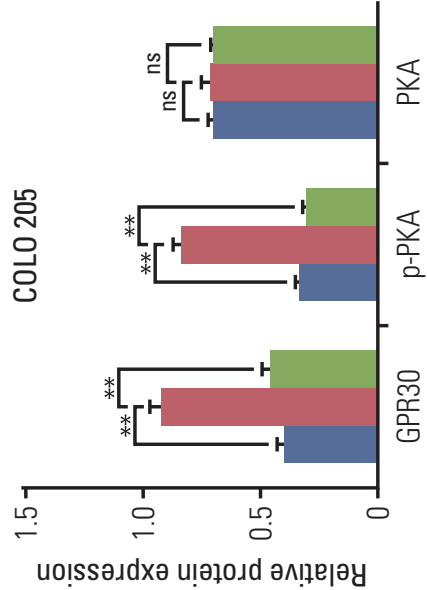

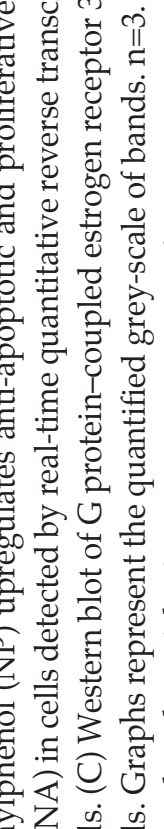

芯出宁

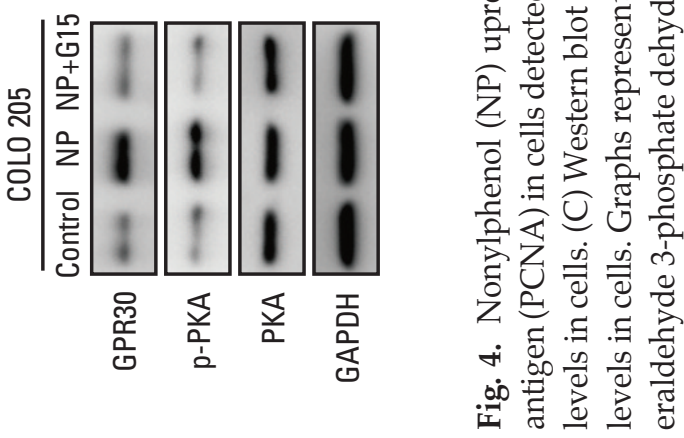



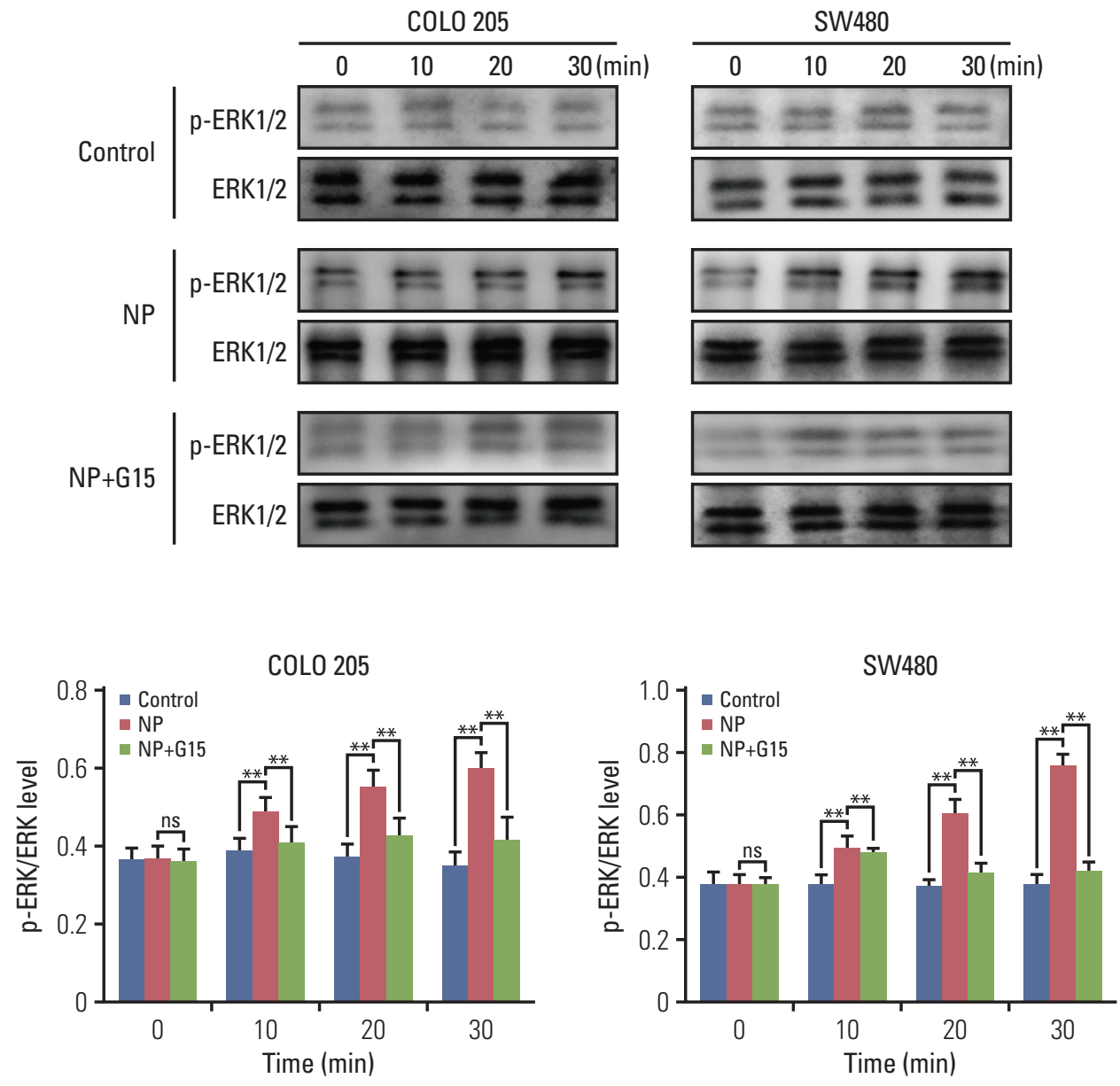

Fig. 5. Nonylphenol (NP) activates ERK1/2 signaling in colon cancer cells. (A) Western blot of phosphorylated ERK1/2 ( $\mathrm{p}$-ERK1/2) and total ERK1/2 proteins in COLO 205 cells. Graph represents the quantified grey-scale of bands. $\mathrm{n}=3$. (B) Significant difference compared to the control group $\left({ }^{* *} \mathrm{p}<0.01\right)$. ns, not significant.

\section{Ethical statement}

All experiments were conducted in accordance with the ethical guidelines and protocols were approved by the Animal Care and Use Committee of the Zunyi Medical College.

\section{Results}

\section{NP promotes cell proliferation and apoptosis failure in colon cancer cells}

To investigate the effects of NP on colon carcinoma in vitro, the colon cancer cell line COLO 205 and the colon adenocar- cinoma cell line SW480 were exposed to treatment with NP or E2, ranging from $10^{-7}$ to $10^{-5} \mu \mathrm{M}$. MTT assay revealed that E2 selectively reduced the proliferation rate of colon cancer cells, as cell viability was unchanged in SW480 cells treated with E2 (Fig. 1A). Meanwhile, NP dose-dependently increased cell viability of both cell lines. Next, flow cytometry was employed to assess apoptosis. As shown in Fig. 1B, E2 dramatically increased the rate of apoptosis in COLO 205 cells, but no significant alteration was observed in SW480 cells. However, NP significantly reduced the ratio of apoptotic cells. Taken together, these data suggest that NP facilitates cell proliferation and suppresses apoptosis in colon cancer cells. 
A
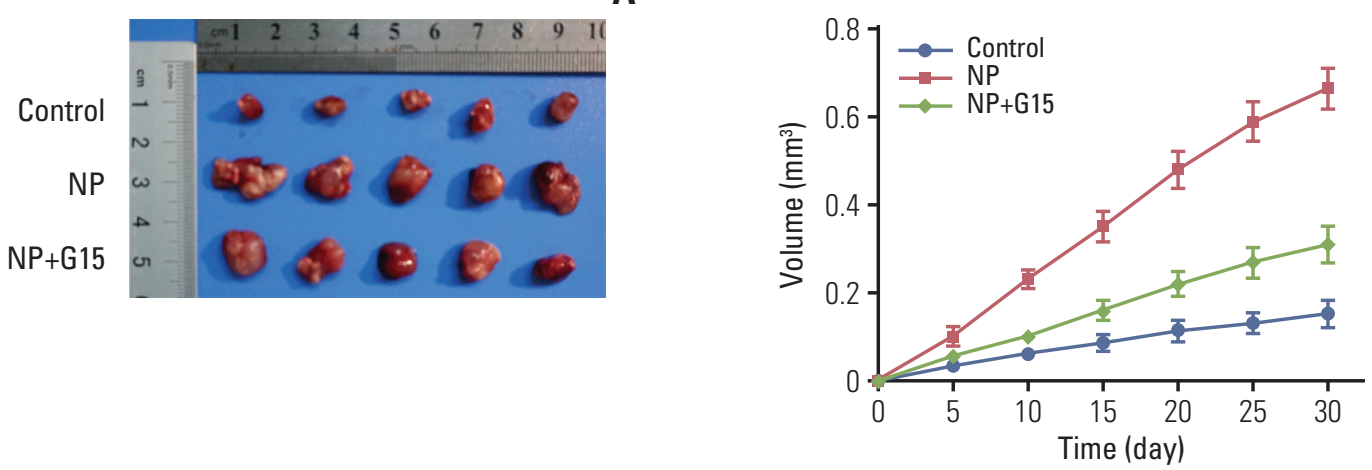

C
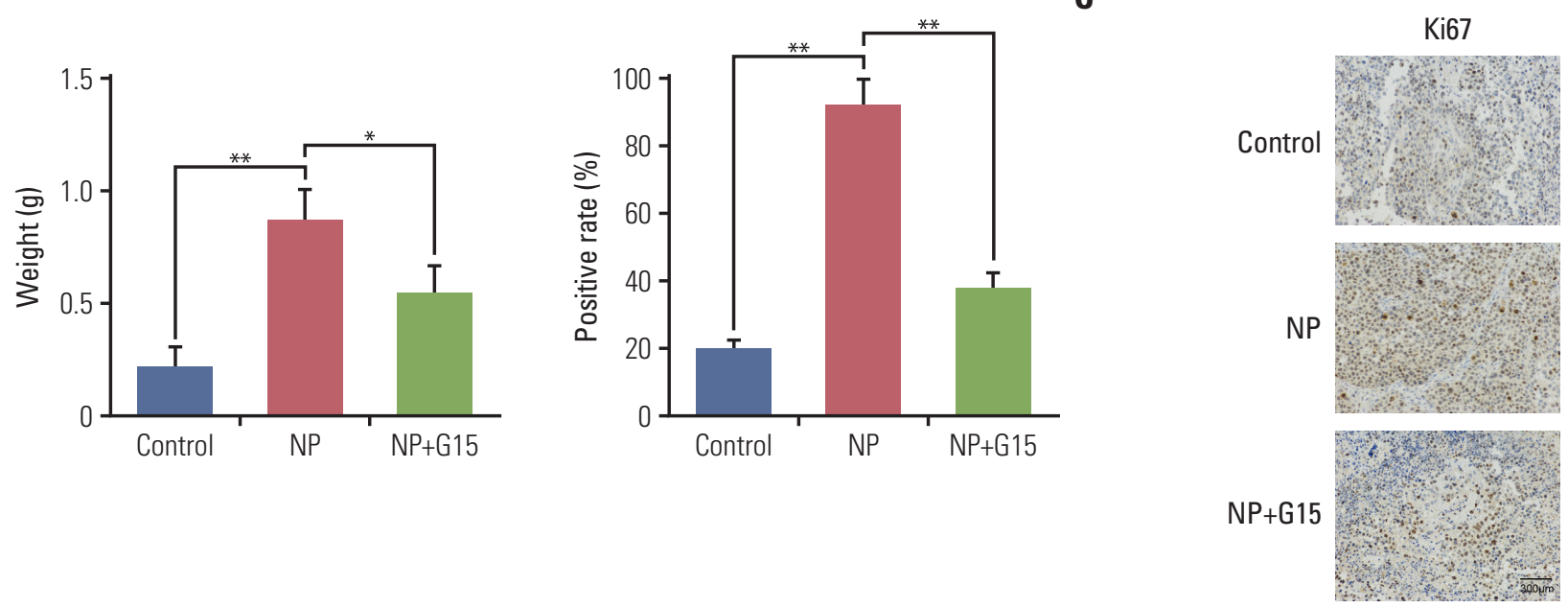

Fig. 6. Nonylphenol (NP) accelerates tumor growth of colon carcinoma in vivo. (A) Representative images of tumors from xenografts receiving various treatments for 15 days. (B) Tumor volume was calculated every 5 days after injection, and tumors were dissected after 30 days. (C) Weight of tumors harvested from mice at the end of experiments $\left({ }^{*} \mathrm{p}<0.05,{ }^{* *} \mathrm{p}<\right.$ 0.01). (D) Immunohistochemistry staining of Ki67. Scale bar $=300 \mu \mathrm{m}$.

\section{NP-mediated cell proliferation is GPR30-dependent}

Environmental estrogens demonstrate relatively high binding affinities for the seven-transmembrane receptor GPR30 compared to classical estrogen receptors [14]. Furthermore, GPR30 has been implicated in regulating cell proliferation and invasion in numerous cancers $[15,16]$. Thus, we hypothesized that GPR30 participates in NP-mediated proliferation. Immunocytochemistry staining revealed that in COLO 205 and SW480 cells, no obvious changes occurred in GPR30 expression in the E2 treatment group (Fig. 2A). In contrast, NP significantly enhanced the expression level of GPR30, as shown by increased red fluorescence intensity of cells membranes. Next, total protein was extracted, and the expression level of GPR30 was assessed using Western blot. Consistent with the immunofluorescence results, Western blot demonstrated that NP increases the GPR30 expression level (Fig. 2B).

Next, G15, a GPR30 antagonist, was employed to confirm the role of GPR30 in colon cancer cells receiving NP treatment [17]. In COLO 205 cells, NP-induced cell proliferation was reversed by treatment with G15 (Fig. 3A), and similar results were observed in SW480 cells. Next, the colony formation efficiencies of COLO 205 and SW480 were evaluated using soft agar colony formation assay. NP significantly increased the colony formation capacity of colon cancer cells, which was dramatically inhibited in the G15 group. Moreover, NP-induced apoptosis failure was abrogated by G15 (Fig. 3C). Taken together, NP boosted the proliferation of colon cancer cells through activating GPR30, thereby protecting cancer cells from apoptosis. 


\section{NP regulates the proliferation of colon cancer cells through ERK1/2 signaling}

To unravel the mechanism by which NP-stimulated GPR30 regulates cell proliferation in colon cancer cells, we examined the effects of GPR30 on c-myc, a transcription factor that regulates tumorigenesis. mRNA levels of c-myc, proliferation marker PCNA, and cyclin D1 were significantly upregulated in response to NP treatment (Fig. 4A). Co-treatment of $\mathrm{NP}+\mathrm{G} 15$ caused the NP-induced upregulation of the proteins mentioned above back to basal levels. The Western blot results were consistent with that of qRT-PCR (Fig. 4B). To assess the significance of GPR30 and p-PKA in NP-induced proliferation, we treated cells with either NP or NP+G15. The results showed that GPR30 and p-PKA increased in NP-challenged cells and decreased in NP+G15-treated cells when compared to the NP group.

Previous studies reported that GPR30 promotes the growth of Sertoli TM4 cell through activation of ERK1/2 [18]. Next, we assessed the activation of ERK signaling and discovered that the ratio of $\mathrm{p}$-ERK/ERK was remarkably increased by NP (Fig. 5A and B). Our results indicate that NP mediates the proliferation of colon cancer cells through the activation of ERK1/ 2 signaling.

\section{NP facilitates tumor growth of colon carcinoma in vivo}

To evaluate the effects of NP in colon carcinoma tumorigenesis in vivo, COLO 205 cells were subcutaneously injected into nude mice. Mice were divided into control, NP treatment, and NP+G15 groups. At the end of 14-day treatments, animals were sacrificed, and tumors were excised. The results revealed that compared to the control group, the tumor size, volume, and weight were significantly inhibited by G15 treatment (Fig. 6A-C). Next, we investigated the expression of Ki67 in tumor tissues. Immunohistochemical data demonstrated that NP-induced increases in Ki67 were significantly suppressed by G15 (Fig. 6D). Taken together, these data suggest that NP promotes colon cancer cell proliferation in a GPR30-dependent manner.

\section{Discussion}

GPR30 is widely expressed in various tissues and cells, including lung, liver, prostate, and ovary. In addition, GPR30 is highly expressed in numerous estrogen relevant diseases and plays a role in E2-mediated efficacy, for example, in promoting cell proliferation, cell metastasis and enhancing apoptosis [2,14,19]. Arias-Pulido et al. [20] analyzed the expression of GPR30 in 88 primary inflammatory breast cancer patients and found that $69 \%$ of them were GPR30 positive. Co-expression of GRP30 and ER is associated with improved overall survival or disease-free survival. A significant increase in GPR30 expression was also reported in human non-small cell lung cancer cell lines compared to immortalized normal lung bronchial epithelial cells [21]. He et al. [22] reported that GPR30 mediates the invasion and carcinogenesis of endometrial cancer cell line RL95-2 through mitogen-activated protein kinase (MARK) activation. These findings suggest that GPR30 mediates the development of numerous tumors, and direct modulation of GPR30 might be a novel strategy for cancer treatment.

In the present study, we report that NP increases proliferation of COLO 205 and SW480 cells. Since ER $\alpha$ is absent in COLO 205 cells and endogenous ER $\beta$ was not detectable in SW480 cells, NP may exert its function independent of classic ERs [23]. Traditionally, the gene regulation effects of oestrogenic activities have been ascribed to two nuclear hormone receptors, ER $\alpha$ and ER $\beta$ [24]. However, over the past decades, a seven-transmembrane $G$ protein-coupled receptor, GPR30, has been discovered as a novel mechanism of estrogenmediated rapid cellular signaling $[2,25]$. The affinity of E2 and GPR30 is 10 times greater than that of E2 for ER $\alpha$, and GPR30 mediates E2-induced nongenomic signaling activities, including adenylyl cyclase stimulation, EGFR transaction, and MAPK activation [16]. In a study of 566 colorectal cancer patients, GPR30 expression was closely associated with poor survival in cancer stages 3-4 in female but not in male patients [26]. Herein, we also observed upregulated expression of GPR30 in colon cancer cells exposed to NP treatment, supporting the protumorigenic role of GPR30 in estrogen-mediated colon carcinoma proliferation and suggesting that intervention with GPR30 antagonists may serve as a novel therapeutic strategy.

NP has been shown to promote the proliferation of breast cancer cells as a consequence of the activation of ER $\alpha$ [27]. However, ER $\beta$ is the predominant estrogen receptor in colon cancer, with limited or no expression of ER $\alpha$ being detected. Expression of ER $\beta$ is down regulated in tissues of patients with colon cancer. Different from GPR30, ER $\beta$ usually conveys protective effects against colon cancer in vivo. NguyenVu et al. [28] elucidated that ER $\beta$ upregulates the expression level of miR-205, which targets oncogenic prospero homebox 1 via its $3^{\prime}$ untranslated region, suppressing the proliferation and metastatic capacity of colon cancer. Hartman et al. [29] demonstrated that ER $\beta$ inhibits colon cancer xenograft growth through cell-cycle modulation. Thus, the combination of ER $\beta$ and GPR30 antagonists would be a promising strategy for colon cancer control.

Taken together, our study indicates that NP affects apoptosis and proliferation of colon cancer through GPR30 and 
ERK1/2 signaling, suggesting that GPR30 is a potential target for colon cancer treatment.

\section{Conflicts of Interest}

Conflict of interest relevant to this article was not reported.

\section{Acknowledgments}

This work was supported by Guizhou Provincial Science and Technology Department (No.Qian Ke He [2017] 1228).

\section{References}

1. Liu MM, Albanese C, Anderson CM, Hilty K, Webb P, Uht $\mathrm{RM}$, et al. Opposing action of estrogen receptors alpha and beta on cyclin D1 gene expression. J Biol Chem. 2002;277: 24353-60.

2. Prossnitz ER, Arterburn JB, Sklar LA. GPR30: a G protein-coupled receptor for estrogen. Mol Cell Endocrinol. 2007;265266:138-42.

3. Filardo EJ, Quinn JA, Frackelton AR Jr, Bland KI. Estrogen action via the $G$ protein-coupled receptor, GPR30: stimulation of adenylyl cyclase and cAMP-mediated attenuation of the epidermal growth factor receptor-to-MAPK signaling axis. Mol Endocrinol. 2002;16:70-84.

4. Kanda N, Watanabe S. 17beta-estradiol inhibits oxidative stress-induced apoptosis in keratinocytes by promoting Bcl-2 expression. J Invest Dermatol. 2003;121:1500-9.

5. Maggiolini M, Vivacqua A, Fasanella G, Recchia AG, Sisci D, Pezzi V, et al. The G protein-coupled receptor GPR30 mediates c-fos up-regulation by 17beta-estradiol and phytoestrogens in breast cancer cells. J Biol Chem. 2004;279:27008-16.

6. Caiazza F, Galluzzo P, Lorenzetti S, Marino M. 17Beta-estradiol induces ERbeta up-regulation via p38/MAPK activation in colon cancer cells. Biochem Biophys Res Commun. 2007;359: 102-7.

7. Barzi A, Lenz AM, Labonte MJ, Lenz HJ. Molecular pathways: estrogen pathway in colorectal cancer. Clin Cancer Res. 2013; 19:5842-8.

8. Wetherill YB, Akingbemi BT, Kanno J, McLachlan JA, Nadal A, Sonnenschein $\mathrm{C}$, et al. In vitro molecular mechanisms of bisphenol A action. Reprod Toxicol. 2007;24:178-98.

9. Fernandez SV, Russo J. Estrogen and xenoestrogens in breast cancer. Toxicol Pathol. 2010;38:110-22.

10. Lee JW, Han HK, Park S, Moon EY. Nonylphenol increases tumor formation and growth by suppressing gender-independent lymphocyte proliferation and macrophage activation. Environ Toxicol. 2017;32:1679-87.

11. Kim SH, Nam KH, Hwang KA, Choi KC. Influence of hexabromocyclododecane and 4-nonylphenol on the regulation of cell growth, apoptosis and migration in prostatic cancer cells. Toxicol In Vitro. 2016;32:240-7.

12. Konstantinopoulos PA, Kominea A, Vandoros G, Sykiotis GP, Andricopoulos P, Varakis I, et al. Oestrogen receptor beta (ERbeta) is abundantly expressed in normal colonic mucosa, but declines in colon adenocarcinoma paralleling the tumour's dedifferentiation. Eur J Cancer. 2003;39:1251-8.

13. Campbell-Thompson M, Lynch IJ, Bhardwaj B. Expression of estrogen receptor (ER) subtypes and ERbeta isoforms in colon cancer. Cancer Res. 2001;61:632-40.

14. Prossnitz ER, Sklar LA, Oprea TI, Arterburn JB. GPR30: a novel therapeutic target in estrogen-related disease. Trends Pharmacol Sci. 2008;29:116-23.

15. He YY, Cai B, Yang YX, Liu XL, Wan XP. Estrogenic G protein-coupled receptor 30 signaling is involved in regulation of endometrial carcinoma by promoting proliferation, invasion potential, and interleukin-6 secretion via the MEK/ERK mitogen-activated protein kinase pathway. Cancer Sci. 2009;100: 1051-61.

16. Kleuser B, Malek D, Gust R, Pertz HH, Potteck H. 17-Betaestradiol inhibits transforming growth factor-beta signaling and function in breast cancer cells via activation of extracellular signal-regulated kinase through the G protein-coupled receptor 30. Mol Pharmacol. 2008;74:1533-43.

17. Chakrabarti S, Davidge ST. G-protein coupled receptor 30 (GPR30): a novel regulator of endothelial inflammation. PLoS One. 2012;7:e52357.

18. Ge LC, Chen ZJ, Liu HY, Zhang KS, Liu H, Huang HB, et al. Involvement of activating ERK1/ 2 through $G$ protein coupled receptor 30 and estrogen receptor $\alpha / \beta$ in low doses of bisphenol A promoting growth of Sertoli TM4 cells. Toxicol Lett. 2014;226:81-9.

19. Mo Z, Liu M, Yang F, Luo H, Li Z, Tu G, et al. GPR30 as an initiator of tamoxifen resistance in hormone-dependent breast cancer. Breast Cancer Res. 2013;15:R114.

20. Arias-Pulido H, Royce M, Gong Y, Joste N, Lomo L, Lee SJ, et al. GPR30 and estrogen receptor expression: new insights into hormone dependence of inflammatory breast cancer. Breast Cancer Res Treat. 2010;123:51-8.

21. Jala VR, Radde BN, Haribabu B, Klinge CM. Enhanced expression of G-protein coupled estrogen receptor (GPER/GPR30) in lung cancer. BMC Cancer. 2012;12:624.

22. He YY, Du GQ, Cai B, Yan Q, Zhou L, Chen XY, et al. Estrogenic transmembrane receptor of GPR30 mediates invasion and carcinogenesis by endometrial cancer cell line RL95-2. J Cancer Res Clin Oncol. 2012;138:775-83.

23. Qiu Y, Waters CE, Lewis AE, Langman MJ, Eggo MC. Oestrogen-induced apoptosis in colonocytes expressing oestrogen receptor beta. J Endocrinol. 2002;174:369-77. 
24. Marino M, Galluzzo P, Ascenzi P. Estrogen signaling multiple pathways to impact gene transcription. Curr Genomics. 2006; 7:497-508.

25. Filardo E, Quinn J, Pang Y, Graeber C, Shaw S, Dong J, et al. Activation of the novel estrogen receptor $G$ protein-coupled receptor 30 (GPR30) at the plasma membrane. Endocrinology. 2007;148:3236-45.

26. Bustos V, Nolan AM, Nijhuis A, Harvey H, Parker A, Poulsom $\mathrm{R}$, et al. GPER mediates differential effects of estrogen on colon cancer cell proliferation and migration under normoxic and hypoxic conditions. Oncotarget. 2017;8:84258-75.
27. Recchia AG, Vivacqua A, Gabriele S, Carpino A, Fasanella G, Rago $\mathrm{V}$, et al. Xenoestrogens and the induction of proliferative effects in breast cancer cells via direct activation of oestrogen receptor alpha. Food Addit Contam. 2004;21:134-44.

28. Nguyen-Vu T, Wang J, Mesmar F, Mukhopadhyay S, Saxena A, McCollum CW, et al. Estrogen receptor beta reduces colon cancer metastasis through a novel miR-205: PROX1 mechanism. Oncotarget. 2016;7:42159-71.

29. Hartman J, Edvardsson K, Lindberg K, Zhao C, Williams C, Strom A, et al. Tumor repressive functions of estrogen receptor beta in SW480 colon cancer cells. Cancer Res. 2009;69:6100-6. 